\title{
Sensitivity analysis of general nonconvex variational inequalities
}

\author{
Muhammad Aslam Noor ${ }^{*}$ and Khalida Inayat Noor
}

"Correspondence:

noormaslam@hotmail.com

Department of Mathematics,

COMSATS Institute of Information

Technology, Park Road, Islamabad,

Pakistan

\begin{abstract}
In this paper, we show that the parametric general nonconvex variational inequalities are equivalent to the parametric Wiener-Hopf equations. We use this alternative equivalent formulation to study the sensitivity analysis for the nonconvex variational inequalities without assuming the differentiability of the given data. Our results can be considered as a significant extension of previously known results for the variational inequalities.
\end{abstract}

MSC: 49J40; 90C33

Keywords: nonconvex variational inequalities; sensitivity analysis; fixed point; Wiener-Hopf equations

\section{Introduction}

Variational inequalities theory, which was introduced by Stampacchia [1], provides us with a simple, natural, general and unified framework to study a wide class of problems arising in pure and applied sciences; see [1-42]. It is well known that the behavior of such problem solutions as a result of changes in the problem data is always of concern. In recent years, much attention has been given to study the sensitivity analysis of variational inequalities. We remark that sensitivity analysis is important for several reasons. First, since estimating problem data often introduces measurement errors, sensitivity analysis helps in identifying sensitive parameters that should be obtained with relatively high accuracy. Second, sensitivity analysis may help to predict the future changes of the equilibrium as a result of changes in the governing systems. Third, sensitivity analysis provides useful information for designing or planning various equilibrium systems. Furthermore, from mathematical and engineering points of view, sensitivity analysis can provide new insights regarding problems being studied and can stimulate new ideas for problem solving. Over the last decade, there has been increasing interest in studying the sensitivity analysis of variational inequalities and variational inclusions. Sensitivity analysis for variational inclusions and inequalities has been studied extensively; see [2, 6, 9-12, 18, 22, 27, 32-34, 38, 40-42]. The techniques suggested so far vary with the problem being studied. Dafermos [6] used the fixed-point formulation to consider the sensitivity analysis of the classical variational inequalities. This technique has been modified and extended by many authors for studying the sensitivity analysis of other classes of variational inequalities and variational inclusions. It is known [39] that the variational inequalities are equivalent to the Wiener-Hopf equations. This alternative equivalent formulation has been used by Noor [18] and Noor

(c) 2013 Noor and Noor; licensee Springer. This is an Open Access article distributed under the terms of the Creative Commons Attribution License (http://creativecommons.org/licenses/by/2.0), which permits unrestricted use, distribution, and reproduction in any medium, provided the original work is properly cited. 
et al. $[32,33]$ to develop the sensitivity analysis framework for various classes of (quasi) variational inequalities.

Noor [31] introduced and considered a new class of variational inequalities on the uniformly prox-regular sets, which are called the general nonconvex variational inequalities. We remark that the uniformly prox-regular sets are nonconvex and include the convex sets as a special case; see $[5,37]$. In this paper, we develop the general framework of sensitivity analysis for the general nonconvex variational inequalities. For this purpose, we first establish the equivalence between parametric general nonconvex variational inequalities and the parametric Wiener-Hopf equations by using the projection technique. This fixedpoint formulation is obtained by a suitable and appropriate rearrangement of the WienerHopf equations. We would like to point out that the Wiener-Hopf equations technique is quite general, unified, flexible and provides us with a new approach to study the sensitivity analysis of nonconvex variational inequalities and related optimization problems. We use this equivalence to develop sensitivity analysis for the nonconvex variational inequalities without assuming the differentiability of the given data. Our results can be considered as significant extensions of the results of Dafermos [6], Moudafi and Noor [12], Noor and Noor $[32,33]$ and others in this area. The ideas and techniques of this paper may stimulate further research in this field.

\section{Preliminaries}

Let $H$ be a real Hilbert space whose inner product and norm are denoted by $\langle\cdot, \cdot\rangle$ and $\|\cdot\|$ respectively. Let $K$ be a nonempty and convex set in $H$.

We, first of all, recall the following well-known concepts from nonlinear convex analysis and nonsmooth analysis [5, 37].

Definition 2.1 The proximal normal cone of $K$ at $u \in H$ is given by

$$
N_{K}^{P}(u):=\left\{\xi \in H: u \in P_{K}[u+\alpha \xi]\right\}
$$

where $\alpha>0$ is a constant and

$$
P_{K}[u]=\left\{u^{*} \in K: d_{K}(u)=\left\|u-u^{*}\right\|\right\} .
$$

Here $d_{K}(\cdot)$ is the usual distance function to the subset $K$, that is,

$$
d_{K}(u)=\inf _{v \in K}\|v-u\| .
$$

The proximal normal cone $N_{K}^{P}(u)$ has the following characterization.

Lemma 2.1 Let $K$ be a nonempty, closed and convex subset in $H$. Then $\zeta \in N_{K}^{P}(u)$ if and only if there exists a constant $\alpha>0$ such that

$$
\langle\zeta, v-u\rangle \leq \alpha\|v-u\|^{2}, \quad \forall v \in K
$$

Definition 2.2 The Clarke normal cone, denoted by $N_{K}^{C}(u)$, is defined as

$$
N_{K}^{C}(u)=\overline{c o}\left[N_{K}^{P}(u)\right]
$$


where $\overline{c o}$ means the closure of the convex hull. Clearly $N_{K}^{P}(u) \subset N_{K}^{C}(u)$, but the converse is not true. Note that $N_{K}^{P}(u)$ is always closed and convex, whereas $N_{K}^{C}(u)$ is convex, but may not be closed $[5,35]$.

Poliquin et al. [37] and Clarke et al. [5] introduced and studied a new class of nonconvex sets, which are called uniformly prox-regular sets. This class of uniformly prox-regular sets has played an important part in many nonconvex applications such as optimization, dynamic systems and differential inclusions.

Definition 2.3 For a given $r \in(0, \infty]$, a subset $K_{r}$ is said to be normalized uniformly $r$-prox-regular if and only if every nonzero proximal normal to $K_{r}$ can be realized by an $r$-ball, that is, $\forall u \in K_{r}$ and $0 \neq \xi \in N_{K_{r}}^{P}(u),\|\xi\|=1$, one has

$$
\langle\xi, v-u\rangle \leq(1 / 2 r)\|v-u\|^{2}, \quad \forall v \in K .
$$

It is clear that the class of normalized uniformly prox-regular sets is sufficiently large to include the class of convex sets, $p$-convex sets, $C^{1,1}$ submanifolds (possibly with boundary) of $H$, the images under a $C^{1,1}$ diffeomorphism of convex sets and many other nonconvex sets; see [5,37]. It is clear that if $r=\infty$, then uniformly prox-regularity of $K_{r}$ is equivalent to the convexity of $K$. It is known that if $K_{r}$ is a uniformly prox-regular set, then the proximal normal cone $N_{K_{r}}^{P}(u)$ is closed as a set-valued mapping. For the sake of simplicity, we take $\gamma=\frac{1}{2 r}$. It is clear that if $r=\infty$, then $\gamma=0$.

For given nonlinear operators $T$, $h$, we consider the problem of finding $u \in H: h(u) \in K_{r}$ such that

$$
\langle\rho T u+h(u)-u, v-h(u)\rangle+\gamma\|v-h(u)\|^{2} \geq 0, \quad \forall v \in K_{r},
$$

where $\rho>0$ and $\gamma>0$ are constants. The inequality of type (1) is called the general nonconvex variational inequality; see Noor [31].

We now discuss some special cases of (1).

(I) If $h \equiv I$, the identity operator, then problem (1) is equivalent to finding $u \in K_{r}$ such that

$$
\langle\rho T u, v-u\rangle+\gamma\|v-u\|^{2} \geq 0, \quad \forall v \in K_{r},
$$

which is known as the nonconvex variational inequality, studied and introduced by Noor [30].

(II) We note that if $K_{r} \equiv K$, the convex set in $H$, then problem (1) is equivalent to finding $u \in H: h(u) \in K$ such that

$$
\langle\rho T u+h(u)-u, v-h(u)| \geq 0, \quad \forall v \in K .
$$

The inequality of type (3) is called the general variational inequality, which was introduced and studied by Noor [29]. 
(III) If $h(u)=u$, then problem (1) is equivalent to finding $u \in H: h(u) \in K_{r}$ such that

$$
\left\langle T(h(u)), v-h(u)+\gamma\|v-h(u)\|^{2}\right\rangle \geq 0, \quad \forall v \in K_{r}
$$

which is also called the general nonconvex variational inequality.

(IV) If $K_{r} \equiv K$, the convex set in $H$, then problem (4) is equivalent to finding $u \in H$ : $h(u) \in K$ such that

$$
\langle T(h(u)), v-h(u)\rangle \geq 0, \quad \forall v \in K
$$

which was introduced and studied by Noor [13] in 1988. It was shown [29] that the minimum of a differentiable nonconvex function can be characterized by general variational inequality (5). See also [20] for its applications in applied sciences.

(V) If $h \equiv I$, the identity operator, then problem (5) is equivalent to finding $u \in K$ such that

$$
\langle T u, v-u\rangle \geq 0, \quad v \in K
$$

which is known as the classical variational inequality introduced and studied by Stampacchia [1] in 1964. It turned out that a number of unrelated obstacle, free, moving, unilateral and equilibrium problems arising in various branches of pure and applied sciences can be studied via variational inequalities; see [1-42] and the references therein.

We now recall the well-known proposition which summarizes some important properties of the uniform prox-regular sets.

Lemma 2.2 Let $K$ be a nonempty closed subset of $H, r \in(0, \infty]$ and set $K_{r}=\{u \in H$ : $d(u, K)<r\}$. If $K_{r}$ is uniformly prox-regular, then

(i) $\forall u \in K_{r}, P_{K_{r}}(u) \neq \emptyset$.

(ii) $\forall r^{\prime} \in(0, r), P_{K_{r}}$ is Lipschitz continuous with constant $\delta=\frac{r}{r-r^{\prime}}$ on $K_{r^{\prime}}$.

(iii) The proximal normal cone is closed as a set-valued mapping.

We now consider the problem of solving the nonlinear Wiener-Hopf equations. To be more precise, let $Q_{K_{r}}=I-h^{-1} P_{K_{r}}$, where $P_{K_{r}}$ is the projection operator, $h^{-1}$ is the inverse of the nonlinear operator $h$ and $I$ is the identity operator. For given nonlinear operators $T, h$, consider the problem of finding $z \in H$ such that

$$
T P_{K_{r}} z+\rho^{-1} Q_{K_{r}} z=0
$$

The equations of type (7) are called general nonconvex Wiener-Hopf equations. Note that if $r=\infty$ and $h=I$, the identity operator, then the nonlinear Wiener-Hopf equations are exactly the same Wiener-Hopf equations associated with variational inequalities (6), which were introduced and studied by Shi [39]. This shows that the original Wiener-Hopf equations are the special case of nonlinear Wiener-Hopf equations (7). The Wiener-Hopf equations technique has been used to study and develop several iterative methods for solving variational inequalities and related optimization problems; see [10-27, 37].

Noor [31] has established the equivalence between general nonconvex variational inequality (1) and the fixed point problem using the projection operator technique. This 
alternative formulation is used to discuss the existence of a solution of problem (1) and to suggest and analyze an iterative method for solving general nonconvex variational inequality (1). For the sake of completeness, we state this result.

Lemma 2.3 [31] $u \in H: h(u) \in K_{r}$ is a solution of (1) if and only if $u \in H: h(u) \in K_{r}$ satisfies the relation

$$
h(u)=P_{K_{r}}[u-\rho T u]
$$

where $P_{K_{r}}$ is the projection of $H$ onto the uniformly prox-regular set $K_{r}$.

Lemma 2.3 implies that general nonconvex variational inequality (8) is equivalent to fixed point problem (8). This alternative equivalent formulation is very useful from the numerical and theoretical point of view.

We now consider the parametric versions of problems (1) and (7). To formulate the problem, let $M$ be an open subset of $H$ in which the parameter $\lambda$ takes values. Let $T(u, \lambda)$ be a given operator defined on $H \times H \times M$ and take value in $H \times H$.

From now onward, we denote $T_{\lambda}(\cdot) \equiv T(\cdot, \lambda)$ unless otherwise specified.

The parametric general nonconvex variational inequality problem is to find $(u, \lambda) \in H \times$ $M$ such that

$$
\left\langle\rho T_{\lambda} u+h(u)-u, v-h(u)\right\rangle \geq 0, \quad \forall v \in K_{r} .
$$

We also assume that for some $\bar{\lambda} \in M$ problem (9) has a unique solution $\bar{u}$.

Related to parametric nonconvex variational inequality (9), we consider the parametric Wiener-Hopf equations. We consider the problem of finding $(z, \lambda) \in H \times M$ such that

$$
T_{\lambda} P_{K_{r}} z+\rho^{-1} Q_{K_{r}} z=0
$$

where $\rho>0$ is a constant and $Q_{K_{r}} z$ is defined on the set of $(z, \lambda)$ with $\lambda \in M$ and takes values in $H$. The equations of type (10) are called the parametric Wiener-Hopf equations.

One can establish the equivalence between problems (9) and (10) by using the projection operator technique; see Noor $[17,18,22]$.

Lemma 2.4 Parametric nonconvex variational inequality (9) has a solution $(u, \lambda) \in H \times M$ if and only if parametric Wiener-Hopf equations (10) have a solution $(z, \lambda) \in H \times M$, where

$$
\begin{aligned}
& h(u)=P_{K_{r}} z, \\
& z=u-\rho T_{\lambda}(u) .
\end{aligned}
$$

From Lemma 2.4, we see that parametric general nonconvex variational inequalities (9) and parametric Wiener-Hopf equations (10) are equivalent. We use this equivalence to study the sensitivity analysis of the general nonconvex variational inequalities. We assume that for some $\bar{\lambda} \in M$ problem (10) has a solution $\bar{z}$ and $X$ is a closure of a ball in $H$ centered at $\bar{z}$. We want to investigate those conditions under which, for each $\lambda$ in a neighborhood of $\bar{\lambda}$, problem (10) has a unique solution $z(\lambda)$ near $\bar{z}$ and the function $z(\lambda)$ is (Lipschitz) continuous and differentiable. 
Definition 2.4 Let $T_{\lambda}(\cdot)$ be an operator on $X \times M$. Then the operator $T_{\lambda}(\cdot)$ is said to be:

(a) Locally strongly monotone if there exists a constant $\alpha>0$ such that

$$
\left\langle T_{\lambda}(u)-T_{\lambda}(v), u-v\right\rangle \geq \alpha\|u-v\|^{2}, \quad \forall \lambda \in M, u, v \in X
$$

(b) Locally Lipschitz continuous if there exists a constant $\beta>0$ such that

$$
\left\|T_{\lambda}(u)-T_{\lambda}(v)\right\| \leq \beta\|u-v\|, \quad \forall \lambda \in M, u, v \in X
$$

\section{Main results}

In this section, we derive the main results of this paper.

We consider the case when the solutions of parametric Wiener-Hopf equations (10) lie in the interior of $X$. Following the ideas of Noor $[17,18,27]$, we consider the map

$$
\begin{aligned}
F_{\lambda}(z) & =P_{K_{r}} z-\rho T_{\lambda}(u), \quad \forall(z, \lambda) \in X \times M \\
& =u-\rho T_{\lambda}(u)
\end{aligned}
$$

where

$$
h(u)=P_{K_{r}} z
$$

We have to show that the map $F_{\lambda}(z)$ has a fixed point, which is a solution of parametric Wiener-Hopf equations (10). First of all, we prove that the map $F_{\lambda}(z)$, defined by (13), is a contraction map with respect to $z$ uniformly in $\lambda \in M$, using essentially the technique of Noor $[17,18,27]$.

Lemma 3.1 Let $P_{K_{r}}$ be a Lipschitz continuous operator with constant $\delta=\frac{r}{r-r^{\prime}}$. Let $T_{\lambda}(\cdot)$ be locally strongly monotone with constant $\alpha>0$ and locally Lipschitz continuous with constant $\beta>0$. If the operator $g$ is strongly monotone with constant $\sigma>0$ and Lipschitz continuous with constant $\delta>0$ respectively then, for all $z_{1}, z_{2} \in X$ and $\lambda \in M$, we have

$$
\left\|F_{\lambda}\left(z_{1}\right)-F_{\lambda}\left(z_{2}\right)\right\| \leq \theta\left\|z_{1}-z_{2}\right\|
$$

where

$$
\begin{aligned}
& \theta=\delta \sqrt{1-2 \alpha \rho+\beta^{2} \rho^{2}}+k, \\
& k=\sqrt{1-2 \sigma+\delta^{2}}
\end{aligned}
$$

for

$$
\begin{aligned}
& \left|\rho-\frac{\alpha}{\beta^{2}}\right|<\frac{\sqrt{\left.\delta^{2} \alpha^{2}-\beta^{2}\left(\delta^{2}-(i-k)^{2}\right)\right)}}{\delta \beta^{2}}, \quad \delta \alpha>\beta \sqrt{\delta^{2}-(1-k)^{2}}, \\
& k=\sqrt{1-2 \sigma+\delta^{2}}<1
\end{aligned}
$$


Proof For all $z_{1}, z_{2} \in X, \lambda \in M$, we have from (13)

$$
\left\|F_{\lambda}\left(z_{1}\right)-F_{\lambda}\left(z_{2}\right)\right\|=\left\|u_{1}-u_{2}-\rho\left(T_{\lambda}\left(u_{1}\right)-T_{\lambda}\left(u_{2}\right)\right)\right\| .
$$

Using the strong monotonicity and Lipschitz continuity of the operator $T_{\lambda}$, we have

$$
\begin{aligned}
& \left\|u_{1}-u_{2}-\rho\left(T_{\lambda}\left(u_{1}\right)-T_{\lambda}\left(u_{2}\right)\right)\right\|^{2} \\
& \quad \leq\left\|u_{1}-u_{2}\right\|^{2}-2 \rho\left\langle T_{\lambda}\left(u_{1}\right)-T_{\lambda}\left(u_{2}\right), u_{1}-u_{2}\right\rangle+\rho^{2}\left\|T_{\lambda}\left(u_{1}\right)-T_{\lambda}\left(u_{2}\right)\right\|^{2} \\
& \quad \leq\left(1-2 \rho \alpha+\rho^{2} \beta^{2}\right)\left\|u_{1}-u_{2}\right\|^{2},
\end{aligned}
$$

where $\alpha>0$ is the strong monotonicity constant and $\beta>0$ is the Lipschitz continuity constant of the operator $T_{\lambda}$ respectively.

From (18) and (19) we have

$$
\left\|F_{\lambda}\left(z_{1}\right)-F_{\lambda}\left(z_{2}\right)\right\| \leq \sqrt{1-2 \alpha \rho+\beta^{2} \rho^{2}}\left\|u_{1}-u_{2}\right\| .
$$

Also from (14) and the Lipschitz continuity of the projection operator $P_{K_{r}}$ with constant $\delta$ we have

$$
\begin{aligned}
\left\|u_{n}-u\right\| & =\left\|u_{n}-u-\left(h\left(u_{n}\right)-h(u)\right)\right\|+\left\|P_{K_{r}} z_{n}-P_{K_{r}} z\right\| \\
& =k\left\|u_{n}-u\right\|+\delta\left\|z_{n}-z\right\|,
\end{aligned}
$$

from which we have

$$
\left\|u_{n}-u\right\| \leq \frac{\delta}{1-k}\left\|z_{n}-z\right\|
$$

Combining (20), (21) and using (15), we have

$$
\begin{aligned}
\left\|F_{\lambda}\left(z_{1}\right)-F_{\lambda}\left(z_{2}\right)\right\| & \leq\left(1-\alpha_{n}\right)\left\|z_{n}-z\right\|+\alpha_{n} \delta \frac{\sqrt{1-2 \rho \alpha+\beta^{2} \rho^{2}}}{1-k}\left\|z_{n}-z\right\| \\
& =\left(1-\alpha_{n}\right)\left\|z_{n}-z\right\|+\alpha_{n} \theta_{1}\left\|z_{n}-z\right\| .
\end{aligned}
$$

From (15) it follows that $\theta<1$ and consequently the map $F_{\lambda}(z)$ defined by (13) is a contraction map and has a fixed point $z(\lambda)$, which is the solution of Wiener-Hopf equation (10).

Remark 3.1 From Lemma 3.1 we see that the map $F_{\lambda}(z)$ defined by (1) has a unique fixed point $z(\lambda)$, that is, $z(\lambda)=F_{\lambda}(z)$. Also, by assumption, the function $\bar{z}$ for $\lambda=\bar{\lambda}$ is a solution of parametric Wiener-Hopf equations (10). Again, using Lemma 3.1, we see that $\bar{z}$ for $\lambda=\bar{\lambda}$ is a fixed point of $F_{\lambda}(z)$ and it is also a fixed point of $F_{\bar{\lambda}}(z)$. Consequently, we conclude that

$$
z(\bar{\lambda})=\bar{z}=F_{\bar{\lambda}}(z(\bar{\lambda}))
$$

Using Lemma 3.1, we can prove the continuity of the solution $z(\lambda)$ of parametric WienerHopf equations (10) using the technique of Noor [17, 18, 22, 27]. However, for the sake of completeness and to convey an idea of the techniques involved, we give its proof. 
Lemma 3.2 Assume that the operator $T_{\lambda}(\cdot)$ is locally Lipschitz continuous with respect to the parameter $\lambda$. If the operator $T_{\lambda}(\cdot)$ is Locally Lipschitz continuous and the map $\lambda \rightarrow P_{K_{r \lambda}} z$ is continuous (or Lipschitz continuous), then the function $z(\lambda)$ satisfying (8) is (Lipschitz) continuous at $\lambda=\bar{\lambda}$.

Proof For all $\lambda \in M$, invoking Lemma 3.1 and the triangle inequality, we have

$$
\begin{aligned}
\|z(\lambda)-z(\bar{\lambda})\| & \leq\left\|F_{\lambda}(z(\lambda))-F_{\bar{\lambda}}(z(\bar{\lambda}))\right\|+\left\|F_{\lambda}(z(\bar{\lambda}))-F_{\bar{\lambda}}(z(\bar{\lambda}))\right\| \\
& \leq \theta\|z(\lambda)-z(\bar{\lambda})\|+\left\|F_{\lambda}(z(\bar{\lambda}))-F_{\bar{\lambda}}(z(\bar{\lambda}))\right\| .
\end{aligned}
$$

From (13) and the fact that the operator $T_{\lambda}$ is Lipschitz continuous with respect to the parameter $\lambda$, we have

$$
\begin{aligned}
\left\|F_{\lambda}(z(\bar{\lambda}))-F_{\bar{\lambda}}(z(\bar{\lambda}))\right\| & =\left\|u(\bar{\lambda})-u(\bar{\lambda})+\rho\left(T_{\lambda}(u(\bar{\lambda}), u(\bar{\lambda}))-T_{\bar{\lambda}}(u(\bar{\lambda}), u(\bar{\lambda}))\right)\right\| \\
& \leq \rho \mu\|\lambda-\bar{\lambda}\| .
\end{aligned}
$$

Combining (22) and (23), we obtain

$$
\|z(\lambda)-z(\bar{\lambda})\| \leq \frac{\rho \mu}{1-\theta}\|\lambda-\bar{\lambda}\| \quad \text { for all } \lambda, \bar{\lambda} \in M,
$$

from which the required result follows.

We now state and prove the main result of this paper, which is the motivation our next result.

Theorem 3.1 Let $\bar{u}$ be a solution of parametric general variational inequality (9) and $\bar{z}$ be a solution of parametric Wiener-Hopf equations (10) for $\lambda=\bar{\lambda}$. Let $T_{\lambda}(u)$ be the locally strongly monotone Lipschitz continuous operator for all $u, v \in X$. If the map $\lambda \rightarrow P_{K_{r}}$ is (Lipschitz) continuous at $\lambda=\bar{\lambda}$, then there exists a neighborhood $N \subset M$ of $\bar{\lambda}$ such that for $\lambda \in N$ parametric Wiener-Hopf equations (10) have a unique solution $z(\lambda)$ in the interior of $X, z(\bar{\lambda})=\bar{z}$ and $z(\lambda)$ is (Lipschitz) continuous at $\lambda=\bar{\lambda}$.

Proof Its proof follows from Lemmas 3.1, 3.2 and Remark 3.1.

\section{Conclusion}

In this paper, we have shown that the parametric general nonconvex variational inequalities are equivalent to the parametric nonconvex Wiener-Hopf equations. These equivalent formulations have been used to develop the general framework of the sensitivity analysis of the general nonconvex variational inequalities without assuming the differentiability of the given data. We expect that the ideas and techniques of this paper will motivate and inspire the interested readers to explore their novel and other applications in various fields.

The authors declare that they have no competing interests. 


\section{Acknowledgements}

The authors would like to express their sincere gratitude to Dr. M. Junaid Zaidi, Rector, CIIT, for providing excellent research facilities

Received: 6 December 2012 Accepted: 16 June 2013 Published: 1 July 2013

\section{References}

1. Stampacchia, G: Formes bilineaires coercitives sur les ensembles convexes. C. R. Math. Acad. Sci. Paris $258,4413-4416$ (1964)

2. Agarwal, RP, Cho, JJ, Huang, NJ: Sensitivity analysis for strongly nonlinear quasi variational inclusions. Appl. Math. Lett. 13, 19-24 (2000)

3. Baiocchi, C, Capelo, A: Variational and Quasi Variational Inequalities. Wiley, London (1984)

4. Bounkhel, M, Tadji, L, Hamdi, A: Iterative schemes to solve nonconvex variational problems. J. Inequal. Pure Appl. Math. 4, 1-14 (2003)

5. Clarke, FH, Ledyaev, YS, Wolenski, PR: Nonsmooth Analysis and Control Theory. Springer, Berlin (1998)

6. Dafermos, S: Sensitivity analysis in variational inequalities. Math. Oper. Res. 13, 421-434 (1988)

7. Giannessi, F, Maugeri, A: Variational Inequalities and Network Equilibrium Problems. Plenum, New York (1995)

8. Kinderlehrer, D, Stampacchia, G: An Introduction to Variational Inequalities and Their Applications. SIAM, Philadelphia (2000)

9. Kyparisis, J: Sensitivity analysis framework for variational inequalities. Math. Program. 38, 203-213 (1987)

10. Kyparisis, J: Sensitivity analysis for variational inequalities and nonlinear complementarity problems. Ann. Oper. Res. 27, 143-174 (1990)

11. Liu, J: Sensitivity analysis in nonlinear programs and variational inequalities via continuous selections. SIAM J. Control Optim. 33, 1040-1068 (1995)

12. Moudafi, A, Noor, MA: Sensitivity analysis for variational inclusions by Wiener-Hopf equations technique. J. Appl. Math. Stoch. Anal. 12, 223-232 (1999)

13. Lions, JL, Stampacchia, G: Variational inequalities. Commun. Pure Appl. Math. 20, 493-512 (1967)

14. Noor, MA: On Variational Inequalities. Ph.D. thesis, Brunel University, London, UK (1975)

15. Noor, MA: General variational inequalities. Appl. Math. Lett. 1, 119-121 (1988)

16. Noor, MA: Quasi variational inequalities. Appl. Math. Lett. 1, 367-370 (1988)

17. Noor, MA: Wiener-Hopf equations and variational inequalities. J. Optim. Theory Appl. 79, 197-206 (1993)

18. Noor, MA: Sensitivity analysis for quasi variational inequalities. J. Optim. Theory Appl. 95, 399-407 (1997)

19. Noor, MA: Some recent advances in variational inequalities. Part. I. Basic concepts. N.Z. J. Math. 26, 53-80 (1997)

20. Noor, MA: Some recent advances in variational inequalities. Part II. Other concepts. N.Z. J. Math. 26, 229-255 (1997)

21. Noor, MA: New approximation schemes for general variational inequalities. J. Math. Anal. Appl. 251, 217-229 (2000)

22. Noor, MA: Some developments in general variational inequalities. Appl. Math. Comput. 152, 199-277 (2004)

23. Noor, MA: Iterative schemes for nonconvex variational inequalities. J. Optim. Theory Appl. 121, 385-395 (2004)

24. Noor, MA: Fundamentals of mixed quasi variational inequalities. Int. J. Pure Appl. Math. 15, 137-258 (2004)

25. Noor, MA: Fundamentals of equilibrium problems. Math. Inequal. Appl. 9, 529-566 (2006)

26. Noor, MA: Differentiable nonconvex functions and general variational inequalities. Appl. Math. Comput. 199, 623-630 (2008)

27. Noor, MA: Extended general variational inequalities. Appl. Math. Lett. 22, 182-185 (2009)

28. Noor, MA: Some iterative methods for general nonconvex variational inequalities. Math. Comput. Model. 54, 2955-2961 (2011)

29. Noor, MA: On a class of general variational inequalities. J. Adv. Math. Stud. 1, 75-86 (2008)

30. Noor, MA: Projection methods for nonconvex variational inequalities. Optim. Lett. 3, 411-418 (2009)

31. Noor, MA: Variational inequalities and applications. Lecture Notes. COMSATS Institute of Information Technology. Islamabad (2008-2013)

32. Noor, MA, Noor, Kl: Sensitivity analysis for quasi variational inclusions. J. Math. Anal. Appl. 236, 290-299 (1999)

33. Noor, MA, Noor, Kl: Sensitivity analysis of some quasi variational inequalities. J. Adv. Math. Stud. 6(1), 43-52 (2013)

34. Noor, MA, Noor, Kl: Some new classes of quasi split feasibility problems. Appl. Math. Inf. Sci. 7(4), 1544-1552 (2013)

35. Noor, MA, Noor, Kl, Al-Said, E: Iterative methods for solving nonconvex equilibrium variational inequalities. Appl. Math. Inf. Sci. 6(1), 65-69 (2012)

36. Noor, MA, Noor, Kl, Rassias, TM: Some aspects of variational inequalities. J. Comput. Appl. Math. 47, 285-312 (1993)

37. Poliquin, RA, Rockafellar, RT, Thibault, L: Local differentiability of distance functions. Trans. Am. Math. Soc. 352, $5231-5249(2000)$

38. Qiu, Y, Magnanti, TL: Sensitivity analysis for variational inequalities defined on polyhedral sets. Math. Oper. Res. 14 410-432 (1989)

39. Shi, P: Equivalence of Wiener-Hopf equations with variational inequalities. Proc. Am. Math. Soc. 111, 339-346 (1991)

40. Tobin, RL: Sensitivity analysis for variational inequalities. J. Optim. Theory Appl. 48, 191-204 (1986)

41. Yen, ND: Holder continuity of solutions to a parametric variational inequality. Appl. Math. Optim. 31, 245-255 (1995)

42. Yen, ND, Lee, GM: Solution sensitivity of a class of variational inequalities. J. Math. Anal. Appl. 215, 46-55 (1997)

doi:10.1186/1029-242X-2013-302

Cite this article as: Noor and Noor: Sensitivity analysis of general nonconvex variational inequalities. Journal of Inequalities and Applications 2013 2013:302. 